\title{
Epidemiologia de Oestrus Ovis (Diptera: Oestridae) em ovinos no Planalto Catarinense
}

\author{
Epidemiology of Oestrus ovis (Diptera: Oestridae) in sheep at the Santa Catarina State Plateau, Brazil
}

\author{
César Itaqui Ramos ${ }^{1}$ Valdomiro Bellato ${ }^{2}$ Antonio Pereira de Souza ${ }^{2}$ Volney Silveira de Avila ${ }^{1}$ \\ Guilherme Caldeira Coutinho $^{1}$ Celso Augustinho Dalagnol $^{1}$
}

\section{RESUMO}

Com os objetivos de determinar a prevalência, a intensidade de infestação e a variação sazonal de larvas de Oestrus ovis, em ovinos, no Planalto Catarinense e propor um esquema estratégico de controle, de outubro de 1997 a setembro de 2001, foi desenvolvido este trabalho em três propriedades rurais nos municípios de Lages, São Joaquim e Campos Novos - SC, Brasil. Para isso, foram utilizados mensalmente três ovinos traçadores por propriedade, os quais, antes de serem conduzidos às mesmas, foram estabulados por 30 dias e executados tratamentos supressivos com diferentes princípios ativos. A seguir, foram encaminhados às três propriedades onde permaneceram em pastejo por 28 dias, sendo posteriormente recolhidos ao estábulo por mais 20 dias. Após, foram sacrificados e coletadas as larvas de $\boldsymbol{O}$. ovis, nos seios paranasais. Quando a temperatura média mensal foi inferior a 9,8 $8^{\circ}$ não se observou animais com larvas de $\boldsymbol{O}$. ovis. Verificou-se que as maiores infestações ocorreram na primavera e verão, principalmente nos municípios de Lages e Campos Novos. Com base nesses resultados, recomenda-se avaliar esquema de controle estratégico no início da primavera e final do verão.

Palavras-chave: Oestrus ovis, epidemiologia, ovinos.

\section{ABSTRACT}

An experiment has been carried out in the Santa Catarina Plateau to recommend a proposal for a strategy of Oestrus ovis, infestation in sheep. The prevalence, intensity and seasonal variation of $O$. ovis larvae in three farms of Lages, São Joaquim, and Campos Novos municipalities in the Santa Catarina State, Brazil, were assessed from october 1997 to september 2001. Three tracer lambs per property were used monthly. They had been previously housed for 30 days and suppressively treated with different active ingredients and taken to their respective farms, where they grazed for 28 days and housed again for another 20 days. After this period, they were sacrificed to collected $O$. ovis larvae from paranasal sinus. No infested animals with larvae of $O$. ovis were observed at monthly average temperatures inferior to $9.8^{\circ} \mathrm{C}$. Heavier infestations occurred in spring and summer, mainly in Lages and in Campos Novos. These results suggest that strategic treatment should be done in the beginning of spring and in the end of summer.

Key words: Oestrus ovis, epidemiology, sheep.

\section{INTRODUÇÃO}

O estado de Santa Catarina possui um rebanho ovino estimado em 202.694 cabeças, com características voltadas principalmente para a produção de carnes; o sistema de criação é intensivo ou semiintensivo. Este rebanho está distribuído, praticamente, em todos os municípios, com predominância na Região Serrana (38,70\%) e Oeste Catarinense (32,79\%), (IBGE, 1997).

Dentre as parasitoses que interferem no sistema produtivo de ovinos em Santa Catarina, está a oestrose, enfermidade parasitária produzida pelas larvas da mosca Oestrus ovis. O parasitismo por esta espécie cosmopolita é comum, tal como registraram MORSY et al. (1991), AARTSEN \& ZEEGERS (1992), BIU \& NWOSU (1998) e DORCHIES et al. (1999).

Nas condições climáticas do Oeste da Europa, YILMA \& DORCHIES (1991) reportaram que, adultos de $\boldsymbol{O}$. ovis foram ativos durante a primavera,

\footnotetext{
${ }^{1}$ Empresa de Pesquisa Agropecuária e Extensão Rural de Santa Catarina S. A. - EPAGRI, CP 181, CEP. 88.502-970, Lages, SC., Brasil. E-mail: itaqui@epagri.rct-sc.br. Autor para correspondência.

${ }^{2}$ Departamento de Med. Vet. Preventiva e Tecnologia - Centro de Ciências Agroveterinárias - CAV. Universidade do Estado de Santa Catarina - UDESC, Lages, SC, Brasil.
} 
verão e outono, e que, no Sul da França, o desenvolvimento do primeiro e segundo ínstares iniciou em fevereiro. Porém, quando as condições climáticas são desfavoráveis, principalmente baixas temperaturas e alta percentagem de umidade relativa pode ocorrer o fenômeno de diapausa, considerado quando se verifica a presença de mais de $80 \%$ de $\mathrm{L}_{1}$ sobre o total da carga de larvas (DORCHIES \& ALZIEU, 1997).

SCALA et al. (2002), na região da Sardenha, Itália, comprovaram que larvas de $1^{\circ}$ estádio $\left(\mathrm{L}_{1}\right)$ entram em diapausa no período de outubro a fevereiro $(80 \%)$. A fase ativa do ciclo endógeno

No Brasil, FREIRE(1958 e 1967)e OLIVEIRA \& MELLO (1981) registraram no Rio Grande do Sul, a presença de larvas da mosca $\boldsymbol{O}$. ovis parasitando ovinos e caprinos, respectivamente. OLIVEIRA et al. (1976/ 1977) constataram uma prevalência em torno de $90 \%$ em ovinos parasitados provenientes do município de Mostardas, RS. Da mesma forma, RIBEIRO et al. (1990), em Bagé, RS, estudaram a prevalência e variação mensal de larvas de $\boldsymbol{O}$. ovis em ovinos, e encontraram uma prevalência de $85,4 \%$ com as maiores intensidades de infestação nos meses de abril e julho. Os piques populacionais das larvas de $1^{\circ}$ e $2^{\circ}$ ínstares ocorreram nos meses de abril e julho e do $3^{\circ}$ ínstar, nos meses de fevereiro, julho e outubro.

A necessidade de conhecer a epidemiologia da espécie $\boldsymbol{O}$. ovis no Planalto Catarinense, que possibilite a indicação de um programa de controle estratégico para os ovinos, foi o principal objetivo do presente trabalho.

\section{MATERIAL E MÉTODOS}

Mensalmente, durante outubro de 1997 a setembro de 2001, um grupo de nove ovinos, foi estabulado durante 30 dias para realização de três tratamentos supressivos, a cada sete dias, na seguinte seqüência: ivermectina, levamisole e triclorfon, de tal forma que o poder residual não ultrapassasse ao período de estabulação. Logo após esta fase, estes animais, chamados traçadores, foram conduzidos, no início de cada mês, três para cada uma das propriedades rurais representativas da região, com criação de ovinos junto com bovinos, nos municípios de Lages $\left(27^{\circ} 48^{\prime} 05^{\prime \prime} \mathrm{S}\right.$, $50^{\circ} 20^{\prime} 06^{\prime \prime} \mathrm{W}$ e altitude $960 \mathrm{~m}$ ), Campos Novos (27 22'59"' $\mathrm{S}, 51^{\circ} 12^{\prime} 55^{\prime \prime} \mathrm{W}$ e altitude $\left.964 \mathrm{~m}\right)$ e São Joaquim ( $28^{\circ}$ $17^{\prime} 39^{\prime}$ ' S, $49^{\circ} 55^{\prime} 56^{\prime}$ ' W e altitude $1415 \mathrm{~m}$ ), no Planalto Catarinense, onde permaneceram por 28 dias em pastejo junto com os demais ovinos das propriedades. Posteriormente, retornaram ao estábulo por mais 20 dias antes de serem abatidos. Destes animais também foram coletados outros parasitos para estudo da epidemiologia de helmintos gastrintestinais (RAMOS et al. 2004). Para as avaliações do presente trabalho, foi realizada a abertura dos seios paranasais para coleta, contagem e posterior identificação dos estágios larvais de $\boldsymbol{O}$. ovis, de acordo com os caracteres descritos por ZUMPT (1965).

\section{RESULTADOS E DISCUSSÃO}

A metodologia utilizada, possibilitou verificar a intensidade de infestação por larvas de $\boldsymbol{O}$. ovis que os ovinos traçadores adquiriram nos diferentes meses, durante os quatro anos avaliados (Figuras $1 \mathrm{e}$ 2) e relacionar com os dados climáticos (Figura 3). Verificou-se que as mais altas infestações foram de 24 e nove ínstares, nos meses de novembro e dezembro de 2000, respectivamente, em Campos Novos e de 11 em Lages no mês de dezembro deste mesmo ano. Em São Joaquim ocorreu um pico de $10 \mathrm{~L}_{3}$ no mês de julho de 1998 quando a temperatura média do mês foi $9,8^{\circ} \mathrm{C}$. $\mathrm{Na}$ maioria dos demais meses, foram baixos os índices de infestações (Figura 1), resultado esperado, devido ao fato de esses animais permanecerem por somente 30 dias expostos a estas infestações. Durante os quatro anos de avaliação, em 68,75\% dos meses, foram diagnosticados, animais traçadores infestados por larvas de $\boldsymbol{O}$. ovis. Provavelmente a prevalência no rebanho deveria ser bem maior, pois estavam continuamente expostos às infestações.

Quanto à flutuação estacional, verifica-se na Figura 1, que as larvas estão presentes na maioria dos meses do ano, semelhante aos resultados observados por ALCAIDE et al. (2003) no Sudeste da Espanha. As maiores intensidades de infestações foram observadas principalmente nos dois últimos anos, no período de dezembro a abril de 2000 e de novembro de 2000 a março de 2001, em Lages e de junho de 2000 a março de 2001 em Campos Novos, quando as temperaturas médias variaram entre 12 e $20^{\circ} \mathrm{C}$, com tendência de aumento da umidade relativa nesses meses, concordando em parte, com os resultados obtidos no Brasil, no município de Bagé, RS, por RIBEIRO et al. (1990), bem como, em alguns países da Europa, relatados por YILMA \& DORCHIES (1991) e SCALA et al. (2002). As temperaturas mais baixas, combinadas com altas percentagens de umidade relativa, observadas durante os quatro anos em São Joaquim e que difere de Lages e Campos Novos, provavelmente foi determinante no menor estabelecimento das larvas de O.ovis, semelhante ao que descreveram DORCHIES \& ALZIEU (1997)

O que chama a atenção no presente trabalho, é o grau de infestação principalmente por $\mathrm{L}_{3}$ (Figura 2) 


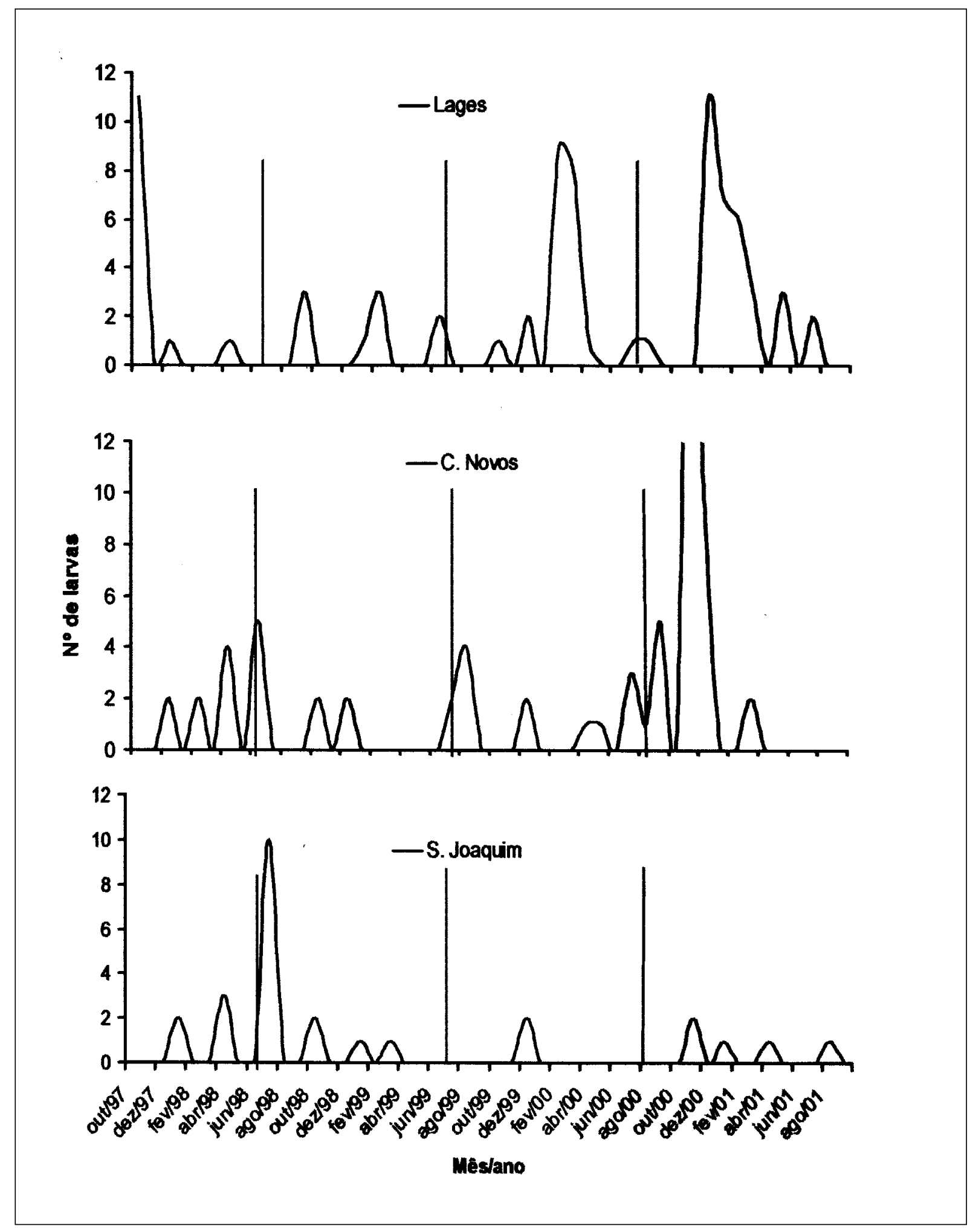

Figura 1 - Flutuação estacional de larvas de Oestrus ovis recuperadas de ovinos traçadores, durante outubro de 1997 a setembro de 2001 , manejados junto a um rebanho de ovinos, nos municípios de Lages, Campos Novos e São Joaquim, SC. 


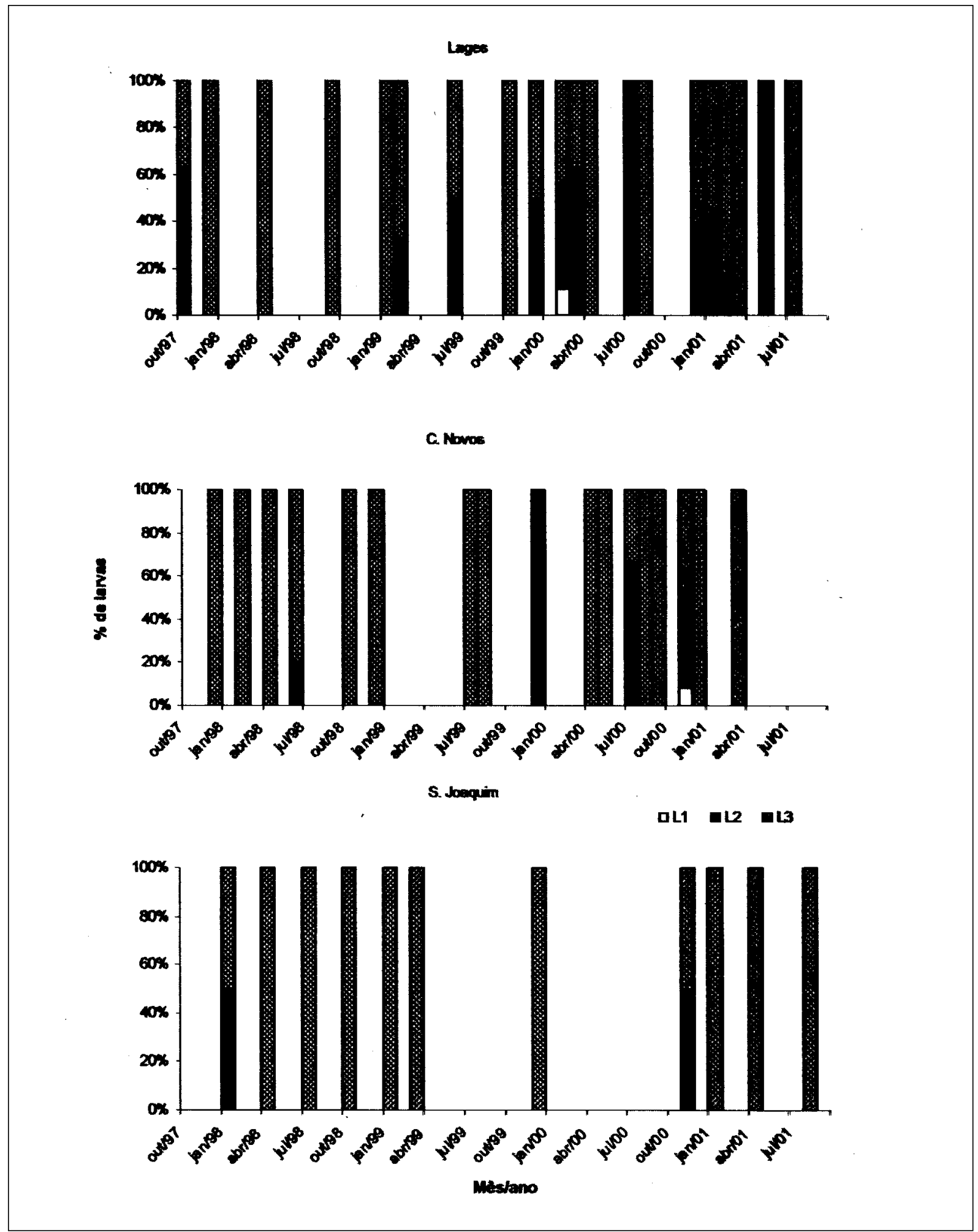

Figura 2 - Percentual de larvas $\left(\mathrm{L}_{1}, \mathrm{~L}_{2}\right.$ e $\left.\mathrm{L}_{3}\right)$ de Oestrus ovis recuperadas de ovinos traçadores, durante outubro de 1997 a setembro de 2001, manejados junto a um rebanho de ovinos, nos municípios de Lages, Campos Novos e São Joaquim, SC.

Ciência Rural, v.36, n.1, jan-fev, 2006. 
sempre presente nos meses de outono e inverno, nos ovinos traçadores, comprovando-se que se tratavam de contaminações adquiridas durante o mês que estiveram junto aos rebanhos, nas três propriedades. No entanto, DORCHIES \& ALZEU (1997) e SCALA et al. (2002), na Itália, região de Sardenha, relataram que a diapausa estava presente durante o outono e inverno (outubro a fevereiro), sem fazer menção sobre a possibilidade de reinfestações nestas épocas do ano.
Verificou-se que as temperaturas médias abaixo de $10^{\circ} \mathrm{C}$ limitaram novas infestações por larvas de $\boldsymbol{O}$. ovis nos animais. A umidade relativa entre $65 \%$ e $90 \%$ com precipitação pluviométrica acima de $100 \mathrm{~mm}$ por mês não foram limitantes para esta mosca durante o período experimental. As $\mathrm{L}_{1}$ foram observadas nos meses de fevereiro em Lages e novembro de $2000 \mathrm{em}$ Campos Novos, as de segundo e terceiro estágio foram encontradas com maior intensidade principalmente na

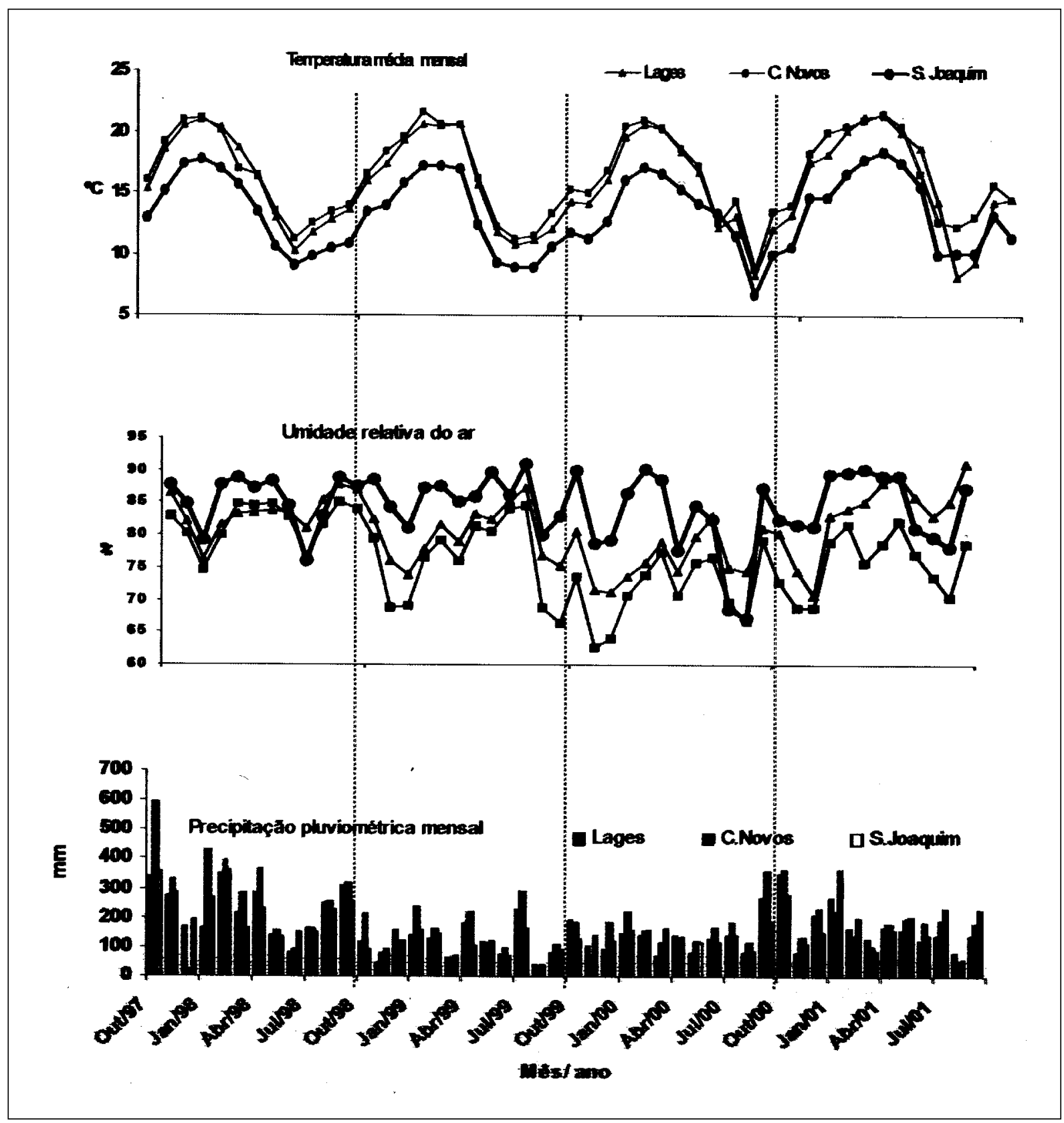

Figura 3 - Dados de clima no período de outubro de 1997 a setembro de 2001, nos municípios de Lages, Campos Novos e São Joaquim, SC.

Ciência Rural, v.36, n.1, jan-fev, 2006. 
primavera e verão nestes municípios (Figura 2). Com base nesses resultados recomenda-se avaliar esquema de controle estratégico medicando os animais no início da primavera e final do verão. Em São Joaquim observou-se $\mathrm{L}_{2}$ apenas nos meses de janeiro de $1998 \mathrm{e}$ novembro de 2000 , não sendo observado $\mathrm{L}_{1}$. Isto provavelmente pode ser explicado pelos baixos índices de infestação durante todo o experimento, limitados pelas temperaturas mais baixas.

\section{CONCLUSÕES}

As larvas de $\boldsymbol{O}$. ovis estão presentes em ovinos, no Planalto Catarinense, com maiores intensidades na primavera e verão. Em temperaturas médias mensais inferiores a $9,8^{\circ} \mathrm{C}$, não foram constatadas larvas de $\boldsymbol{O}$. ovis nos animais.

\section{REFERÊNCIAS}

AARTSEN, B.V.; ZEEGERS, T. De huidige stand van de Nederlands horzelfauna (diptera: Oestridae; Hypodermatidae). Entomologische Berichten, v.52, n.3, p.29-31, 1992.

ALCAIDE, M. et al. Seasonal Variation in the larval burden distribution of Oestrus ovis in sheep in the southwest of Spain Veterinary Parasitology, v.118, n.3/4, p.235-241, 2003.

BIU, A.A.; NWOSU, Ch.O. Oestrus ovis infestation in sheep of semi-arid zone of Nigeria. Pakistan Veterinary Journal, v.18, n.4, p.206-208, 1998.

DORCHIES, P; ALZIEU, J.P. L'oestrose ovine, revue. Révue de Médicine Vétérinaire, v.148, n.7, p.565-574, 1997.

DORCHIES, P. et al. Ostrose du mouton et de la chèvre (Oestrus ovis, Liné 1761) en Afrique: résultats d'une enquête sur 3204 sérums provenant de neuf pays. Révue de Médicine Vétérinaire, v.150, n.5, p.463-466, 1999.
FREIRE, J.J. Fauna zooparasitária rio grandense. Revista da Escola de Agronomia e Veterinária da Universidade Federal do Rio Grande do Sul, v.2, n.1, p.7-43, 1958.

FREIRE, J.J. Fauna parasitária rio grandense. Revista de Medicina Veterinária, v.3, n.1, p.40-55, 1967.

IBGE. Instituto Brasileiro de Geografia e Estatística, Censo Agropecuário, Santa Catarina, 1995-1996. Rio de Janeiro, 1997. 286p.

MORSY, T.A. et al. Some myiasis producers in Cairo and Giza abattoirs. Journal Egypsian of Parasitology, v.21, n.2, p.539546, 1991.

OLIVEIRA, C.M.B. et al. Eficácia do dimetoato no tratamento de Oestrus ovis Lin., 1761. Arquivos da Faculdade de Veterinária, UFRGS, v.4/5, p.89-91, 1976/1977.

OLIVEIRA, C.M.B.; MELLO, J.R.B. Ocorrência de O. ovis L., 1761 parasitando caprinos no Brasil. Arquivos da Faculdade de Veterinária UFRGS, v.9, p.41-42, 1981.

RAMOS, C.I. et al. Epidemiologia das helmintoses gastrintestinais de ovinos no Planalto Catarinense. Ciência Rural, v.34, n.6, p.1889-1895, 2004

RIBEIRO, V.L.S. et al. Prevalência e variações mensais das larvas de Oestrus ovis (Linneus, 1761) em ovinos no município de Bagé, RS, Brasil. Arquivo Brasileiro de Medicina Veterinária e Zootecnia, v.42, n.3, p. 211-221, 1990.

SCALA, A. et al. Chronobiology of Oestrus ovis (Díptera: Oestridae) in Sardinia, Italy: guidelines to chemoprophylaxis. Journal of Medical Entomology, v.39, n.4, p.652-657, 2002.

ZUMPT, F. Myiasis in man and animals in the old world. London: Butterworts, 1965. 267p.

YLMA, J.M.; DORCHIES, Ph. Epidemiology of Oestrus ovis in southwest France. Veterinary Parasitology, v.40. n.3/4, p.315-323, 1991. 